SHORT REPORT

\title{
A case-control study of SARS versus community acquired pneumonia
}

\author{
F W T Cheng, P C Ng, W K Chiu, W C W Chu, A M Li, K L Lo, E K L Hon, E A S Nelson, T F Leung, \\ W H Ng, E Wong, P Ip, T F Fok
}

The clinical, laboratory, and radiological features at presentation of 16 children ( $<12$ years) with severe acute respiratory syndrome (SARS) and pneumonia were compared with 32 age matched patients with community acquired pneumonia for determination of predictive factors that could allow early differentiation of the two conditions. A definitive contact history was the most important predictor for SARS. Raised serum lactate dehydrogenase concentration in the presence of low neutrophil count and serum creatine phosphokinase level at presentation also indicated an increased likelihood of SARS-coronavirus infection in young children.

$\mathrm{D}$ uring the severe acute respiratory syndrome (SARS) outbreak in 2003, a significant number of healthcare workers and in-patients contracted the SARS-coronavirus (SARS-CoV) infection secondary to nosocomial spread in hospital. ${ }^{1}$ An effective triaging strategy for febrile children is important to allow proper segregation of patients and to minimise the chance of spreading the disease. Without an accurate and rapid diagnostic test, paediatricians can only rely on early clinical features and basic laboratory investigations. We compared the clinical, laboratory, and radiological features at presentation in young children with SARS-CoV pneumonia and those with community acquired pneumonia (CAP) for determination of discriminatory factors of the two conditions.

\section{SUBJECTS AND METHODS}

During the SARS outbreak between 13 March 2003 and 17 May 2003 in Hong Kong, 16 children (<12 years) with serologically confirmed SARS-CoV pneumonia were hospitalised; they constituted the index group of the study. Thirty two age matched children diagnosed to have CAP and presenting within the same time period comprised the control group. CAP is defined as (1) the presence of respiratory signs and symptoms, (2) with chest radiological abnormalities compatible with pneumonia, and (3) in a previously healthy child acquiring the respiratory infection in the community. ${ }^{2}$ None of the patients in either group received medical treatment in the preceding 14 days prior to hospital admission. The clinical features at presentation were recorded on a standardised data sheet. These data, together with laboratory and radiological investigations performed immediately after admission are summarised in table 1 .

Throat swab or throat gargle were obtained on admission for (1) antigen detection of influenza A and B, and respiratory syncytial virus (RSV); (ii) isolation of common respiratory viruses, including influenza $\mathrm{A}$ and $\mathrm{B}$, parainfluenza 1, 2, and 3, adenovirus, RSV, and SARS-CoV in specific cell lines; and (3) reverse transcriptase-polymerase chain reaction (RT-PCR) of SARS-CoV. ${ }^{3}$ Paired acute and convalescent serological titres were also taken on admission and 28 days after the onset of fever for respiratory viruses, Mycoplasma pneumoniae, and SARS-CoV. Throat swab and sputum samples were collected for routine bacterial culture. In addition, haematological and biochemical investigations were performed, and included differential white cell counts, lactate dehydrogenase (LDH), alanine aminotransferase, creatine phosphokinase (CPK), clotting profile, and D-dimer. Chest radiograph on admission was taken on day 3 (1-6) [median (range)] after the onset of fever. These radiographs were retrospectively reviewed by designated paediatric radiologists who were blinded to the clinical data of the patients. The method used for chest radiograph assessment had been standardised and was described in detail in an earlier study of adult patients with SARS. ${ }^{4}$ The Mann-Whitney $U$ test and $\chi^{2}$ test were used to compare the clinical, radiological, and laboratory parameters between the groups.

Ethical approval was obtained from the clinical research ethics committee of both participating hospitals for performing the study.

\section{RESULTS}

The median (interquartile range) age of children with SARSCoV pneumonia and CAP was $5.3(2.2-11.0)$ years and 5.8 (3.69.1) years. In the CAP group, 5, 2, 2, and 1 patients had Mycoplasma pneumoniae, Haemophilus influenzae, influenza A, and RSV pneumonia, respectively. There was no significant difference in any of the presenting clinical features between the two groups. Children with SARS-CoV pneumonia had a strong household contact history, and in most cases it was associated with affected family members (OR 42.29 ; 95\% CI 7.17 to $\infty$; $\mathrm{p}<0.0001$ ). Further, higher serum LDH (OR 1.01, 95\% CI 1.01 to $1.02 ; \mathrm{p}<0.0001$ ), and lower neutrophil count (OR 0.64, 95\% CI 0.41 to $0.87 ; \mathrm{p}=0.001$ ) and serum CPK (OR 0.98; $95 \%$ CI 0.97 to $0.99 ; \mathrm{p}=0.01$ ) were significantly more common among SARS children (table 1). A positive contact history and serum LDH concentration $\geqslant 290$ IU/1 ( $14 / 16$ and 4/32 children in SARS and CAP group, respectively) identified all SARS-CoV infected children in the cohort. Table 2 summarises the chest radiological findings on admission; the characteristics of the images were similar between the two groups.

\section{DISCUSSION}

Young children with SARS often presented with non-specific constitutional or respiratory signs and symptoms that were difficult to differentiate from lower respiratory tract infection caused by common respiratory pathogens. Our findings

\footnotetext{
Abbreviations: CAP, community acquired pneumonia; $\mathrm{Cl}$, confidence interval; CPK, creatine phosphokinase; LDH, lactate dehydrogenase; OR, odds ratio; RSV, respiratory syncytial virus; RT-PCR, reverse transcriptase-polymerase chain reaction; SARS, severe acute respiratory syndrome; SARS-CoV, SARS-coronavirus
} 
Table 1 Clinical features and laboratory parameters in SARS and CAP patients on admission

\begin{tabular}{|c|c|c|c|c|}
\hline Clinical features & SARS group $(n=16)$ & CAP group $(n=32)$ & $\begin{array}{l}\text { Matched } \\
\text { odds ratio }\end{array}$ & $95 \% \mathrm{Cl}$ \\
\hline $\begin{array}{l}\text { No. days between onset of } \\
\text { illness and hospital admission }\end{array}$ & $4(2-5)$ & $3(2-3)$ & 1.20 & 0.82 to 1.77 \\
\hline Admission temperature ( $\left.{ }^{\circ} \mathrm{C}\right)$ & $38.8(37.0-39.3)$ & $38.3(37.8-38.7)$ & 1.21 & 0.69 to 2.17 \\
\hline Respiration (/min) & $26(24-30)$ & $25(24-26)$ & 1.09 & 0.91 to 1.32 \\
\hline Chills & $5(31 \%)$ & $14(44 \%)$ & 0.53 & 0.08 to 2.52 \\
\hline Cough & $11(69 \%)$ & $25(78 \%)$ & 0.65 & 0.14 to 2.98 \\
\hline Malaise & $3(19 \%)$ & $9(28 \%)$ & 0.62 & 0.10 to 2.90 \\
\hline Diarrhoea & $3(19 \%)$ & $4(13 \%)$ & 1.83 & 0.18 to 24.10 \\
\hline Household contact history ${ }^{* *}$ & $15(94 \%)$ & $0(0 \%)$ & 42.29 & 7.17 to $+\infty$ \\
\hline Lymphocyte count $\left(\times 10^{9} / \mathrm{I}\right)$ & $1.7(1.2-2.4)$ & $1.3(0.8-2.4)$ & 1.38 & 0.75 to 2.67 \\
\hline Neutrophil count $\left(\times 10^{9} / /\right)^{*}$ & $2.6(1.9-4.7)$ & $6.2(3.9-8.8)$ & 0.64 & 0.41 to 0.87 \\
\hline $\mathrm{LDH}(\mathrm{IU} / \mathrm{I})^{* *}$ & $430(331-562)$ & $270(223-290)$ & 1.02 & 1.01 to 1.03 \\
\hline CPK (IU/I)* & $83(66-119)$ & $119(76-233)$ & 0.98 & 0.97 to 0.99 \\
\hline APTT (sec) & $36(34-39)$ & $36(34-41)$ & 0.96 & 0.81 to 1.12 \\
\hline D-dimer (ng/ml) & $500(500-717)$ & $799(484-1165)$ & 1.00 & 1.00 to 1.00 \\
\hline
\end{tabular}

suggested that a positive household contact history was the most important risk factor for SARS.

Lymphopenia had been reported to be the most frequent haematological abnormality in paediatric and adult SARS patients. ${ }^{15}$ However, a low lymphocyte count was not a good discriminatory factor in young children, as this phenomenon was also commonly observed in CAP at an early phase of the disease (table 1). Neutrophilia was a common finding (82\%) in adult SARS patients, ${ }^{5}$ but in the current study the neutrophil counts in young children tended to be low at presentation. Neutrophilia observed in adult patients was likely to be associated with secondary bacterial infection or the use of high dose corticosteroids, ${ }^{5}$ but such a late complication or treatment would not have occurred in the first few days of illness. In accordance with our findings, recent studies indicated that only a very small proportion of children with SARS $(6.8 \%)$ had neutrophilia at presentation, but many were neutropenic during their illnesses. ${ }^{6}$ Although circulating LDH concentrations in young children with SARSCoV pneumonia were increased above the normal level, they were not as high as those observed in adult SARS patients, especially those complicated by acute respiratory distress syndrome. ${ }^{1}$ Reduced severity of SARS in paediatric patients could explain the moderately increased LDH levels. In this cohort, using a combination of raised serum LDH concentration ( $\geqslant 290 \mathrm{IU} / \mathrm{l})$ and a positive contact history, we were able to identify all children with SARS-CoV pneumonia. Conversely, serum CPK concentrations were paradoxically lower in children with SARS. A plausible explanation is that in most young children, this enzyme did not rise at an early phase of SARS but was already increased in some CAP patients. Chest radiological features on admission did not differ between children with SARS-CoV pneumonia and CAP, illustrating that the radiological abnormalities associated with SARS-CoV were often non-specific and indistinguishable from lower respiratory tract infection due to other common pathogens. Thus, the radiological images could only provide information on the severity of pulmonary involvement but could not be used as a specific tool for diagnosis of SARS.

In summary, in the absence of sophisticated laboratory diagnostic tools such as real time RT-PCR for quantification of SARS-CoV $\mathrm{RNA}^{3}$ in most acute hospitals, a positive household contact history is the most important predictor for SARS in young children. Increased serum LDH in the presence of low neutrophil count and serum CPK at presentation also indicates an increased likelihood of SARS$\mathrm{CoV}$ infection. Radiological images can only provide information on the severity of pulmonary involvement.

\section{Authors' affiliations}

F W T Cheng, P C Ng, A M Li, E K L Hon, E A S Nelson, T F Leung, T F Fok, Department of Paediatrics, Prince of Wales Hospital, The Chinese University of Hong Kong

Table 2 Chest radiological features of SARS and CAP patients on admission

\begin{tabular}{|c|c|c|c|c|}
\hline Chest radiological features & $\begin{array}{l}\text { SARS group } \\
(\mathrm{n}=16)\end{array}$ & $\begin{array}{l}\text { CAP group } \\
(n=32)\end{array}$ & $\begin{array}{l}\text { Matched } \\
\text { odds ratio }\end{array}$ & $95 \% \mathrm{Cl}$ \\
\hline \multicolumn{5}{|l|}{ Radiological abnormalities* } \\
\hline Air space consolidation & $13(81 \%)$ & $19(59 \%)$ & 2.96 & 0.60 to 16.33 \\
\hline Other features (e.g. reticular, nodular) & $0(0 \%)$ & $6(19 \%)$ & & \\
\hline Normal & $3(19 \%)$ & $7(22 \%)$ & & \\
\hline \multicolumn{5}{|l|}{ Number of lesions } \\
\hline Unifocal & $11(69 \%)$ & $17(53 \%)$ & 2.59 & 0.38 to 21.67 \\
\hline Multifocal & $2(13 \%)$ & $8(25 \%)$ & & \\
\hline \multicolumn{5}{|l|}{ Lung volume† } \\
\hline Hyperinflation & $2(13 \%)$ & $13(41 \%)$ & 0.21 & 0.03 to 1.24 \\
\hline Normal volume & $14(88 \%)$ & $18(56 \%)$ & & \\
\hline Volume loss & $0(0 \%)$ & $1(3 \%)$ & & \\
\hline \multicolumn{5}{|l|}{ Distribution of lesions } \\
\hline Peripheral & $6(38 \%)$ & $6(19 \%)$ & 3.43 & 0.68 to 17.95 \\
\hline Central & $7(44 \%)$ & $24(75 \%)$ & & \\
\hline
\end{tabular}

Results are expressed as number (\%).

"OR and 95\% Cl for "air-space consolidation" versus "normal" plus "other features".

†OR and 95\% Cl for "hyperinflation" versus "normal volume" plus "volume loss"

All children in both clinical groups subsequently had abnormal chest radiographs. 
W K Chiu, P Ip, Department of Paediatrics and Adolescent Medicine, United Christian Hospital, Hong Kong

W C W Chu, W H Ng, Department of Diagnostic Radiology and Organ Imaging, The Chinese University of Hong Kong

K L Lo, Department of Radiology and Organ Imaging, United Christian Hospital, Hong Kong

E Wong, Centre of Epidemiology and Biostatistic Research, The Chinese University of Hong Kong

Competing interests: none declared

Correspondence to: Professor P C Ng, Level 6, Department of Paediatrics, Clinical Sciences Building, Prince of Wales Hospital, Shatin, New Territories, Hong Kong; pakcheungng@cuhk.edu.hk

Accepted 4 February 2005

\section{REFERENCES}

1 Lee $\mathrm{N}$, Hui D, Wu A, et al. A major outbreak of severe acute respiratory syndrome in Hong Kong. N Engl J Med 2003;348:1986-94.

2 Pereira JC, Escuder MM. The importance of clinical symptoms and signs in the diagnosis of community-acquired pneumonia. J Trop Pediatr 1998;44:18-24.

$3 \mathrm{Ng}$ EKO, Ng PC, Hon KL, et al. Serial analysis of plasma concentration of SARS coronavirus RNA in pediatric patients with severe acute respiratory syndrome. Clin Chem 2003;49:2085-8.

4 Wong KT, Antonio GE, Hui DSC, et al. Severe acute respiratory syndrome: radiographic appearances and pattern of progression in 138 patients. Radiology 2003;228:401-6.

5 Wong RSM, Wu A, To KF, et al. Haematological manifestations in patients with severe acute respiratory syndrome: retrospective analysis. $B M J$ 2003;326: 1358-62.

6 Bitnun A, Allen U, Heurter $\mathrm{H}$, et al. Children hospitalized with severe acute respiratory syndrome-related illness in Toronto. Pediatrics 2003;112:e261-8.

\section{ARCHIVIST}

\section{Schools and adolescent health}

f

f only we could run schools better then children and adolescents would become more mature, less vulnerable, more socially accomplished, more successful, healthier, and happier people. It's an attractive thought but is it workable? Do school development programmes produce measurable results? The Health Promoting Schools framework was introduced by the World Health Organization in 1995, stressing the quality of the school environment and the importance of community health programmes in schools. Several programmes of school change have been described but they have not been subjected to rigorous objective assessment. The Gatehouse Project in Melbourne, Australia aims to promote the emotional and behavioural wellbeing of adolescents in secondary schools. The results of a randomised controlled trial have been reported (Lyndal Bond and colleagues. Journal of Epidemiology and Community Health 2004;58:997-1003).

The study included 12 intervention and 14 control schools (2678 students) in Melbourne and surrounding districts. In the intervention schools the three priority areas for action were: building a sense of security and trust, increasing communication skills and opportunities, and building a sense of positive regard for the school by participation in aspects of school life. School based adolescent health teams were established, student surveys were performed to identify good and bad factors in the schools' social and learning environments, and strategies were developed using the survey results. Teaching resources were developed to allow students to recognise and tackle social and emotional problems and professional support for teachers was provided by school liaison teams. Data were collected at baseline in school year 8 (mean age 14 years) in 1997 and at the end of school years 8, 9, and 10. Students completed self administered questionnaires using laptop computers. At baseline $53 \%$ of students said they had been bullied, $35 \%$ had low engagement with the school, 18\% had depressive symptoms, 5\% were regular drinkers, $13 \%$ had indulged in binge drinking, $16 \%$ had smoked (but only $2 \%$ regularly), and $7 \%$ had used cannabis. Factors associated with substance abuse included low school engagement, having arguments with many others, and being victimised. There were similar, but stronger, links between social relationships and depressive symptoms.

The intervention had no significant effect on bullying, low school attachment, poor availability of personal attachments, arguments with fellow students, or depressive symptoms. Overall, rates of drinking and smoking were 3-5\% less in intervention schools compared with control schools during the period of follow up. There were significant reductions in regular smoking in the intervention schools.

There was some reduction in smoking in the intervention schools but no significant effect on depressive symptoms, social relationships, or school attachment. The authors conclude that the strategy can be effective in reducing adolescents' health risk behaviour particularly with respect to substance abuse. A barely significant reduction in teenage smoking seems scant reward for much effort but the researchers remain enthusiastic and call for long term commitment by funders, government departments, communities, and schools. Perhaps it takes more than a year or three to change the character of a school. Or perhaps adolescent turmoil and rebelliousness is an essential part of life, susceptible to modification only in part. 